\title{
WORLD HEALTH ORGANIZATION RELEASES INJURY SURVEILLANCE GUIDELINES
}

The World Health Organization's Injury and Violence Prevention Department recently developed a manual, Injury Surveillance Guidlines. The manual is the product of an international collaboration between many agencies, which has drawn on the expertise of staff in the US Centers for Disease Control and Prevention (CDC). The collaboration has benefited from the input of experts from organisations in more than 50 countries, across all continents, who have commented on draft versions of the manual. The work has taken two years to complete.

The aim of the manual is to help people to design, establish, and maintain good injury surveillance systems. It is aimed at researchers and practitioners; and it provides practical advice on how to develop information systems for the systematic collection of data on injuries. Although the manual is applicable to all settings, it has a particular focus on settings where resources are scarce. For such settings, the manual provides practical guidance in how to set up a system to collect, code, and process data, even if there is no electronic equipment and few staff; and/or if staff have other demands on their time or have a greater expertise in research.

As well as a brief overview of its aims and objectives-and an introduction to the terms, analytical tools, and methods used by injury surveillance specialists - the manual takes the reader step-by-step through the process of designing an injury surveillance system. Individual modules for the surveillance of different types of injury at varying levels of detail-from core data through to the optional add-ons-are presented.

For each module, sample forms for recording information on individual cases, and for assembling that information into a dataset, are given. Sample forms for further examples are provided in the appendices, which are referred to as appropriate.

Printed copies of the Injury Surveillance Guidelines can be ordered from the Injuries and Violence Prevention Department, Non-communicable Diseases and Mental Health Cluster, World Health Organization, by mail at 20 Avenue Appia, 1211 Geneva 27, Switzerland; by facsimile at 004122791 4332; or by email at pvi@who.int. A PDF version can be downloaded from www.who.int/violence_injury_prevention/surveillance.htm.

The substance of this notice first appeared in Injury Issues Monitor 24; May 2002: 2. 